\title{
O ensino em grupo do processo de aplicação de insulina
}

\author{
Group teaching of the insulin application process
}

Enseñanza grupal del proceso de aplicación de la insulina

\begin{abstract}
Jéssica Magalhães Felipe Batista ${ }^{1}$, Tânia Alves Canata Becker ${ }^{2}$, Maria Lucia Zanetti ${ }^{3}$, Carla Regina de Souza Teixeira ${ }^{4}$
\end{abstract}

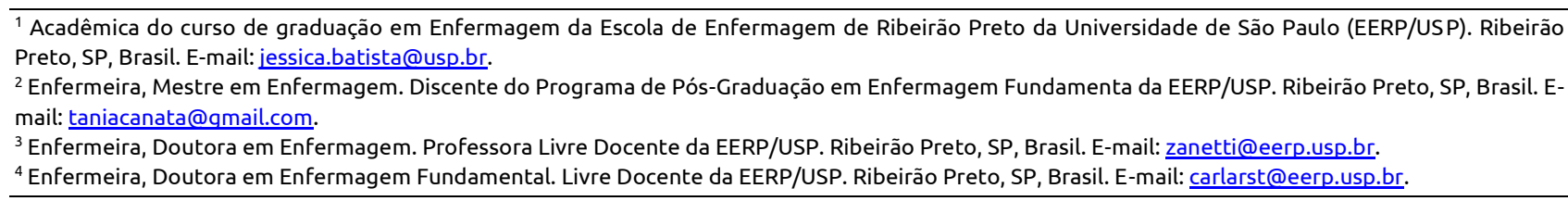

\section{RESUMO}

O presente estudo teve por objetivo analisar a habilidade da pessoa com diabetes mellitus (DM) em realizar o processo de aplicação de insulina antes e após o ensino em grupo desse procedimento. A coleta de dados se deu pela aplicação de um instrumento em forma de check list, que avaliou a habilidade inicial da pessoa com DM em relação ao processo. Posteriormente, foi realizada uma intervenção educativa em grupo, sendo aplicado novamente o mesmo instrumento para avaliação da habilidade final. Os dados obtidos foram armazenados em um banco de dados e submetidos à análise estatística. Os resultados mostraram que a maioria das pessoas apresentou dúvidas em quase todas as etapas do processo de aplicação de insulina, não realizando algumas etapas essenciais ou executando-as de forma errada. Após a intervenção educativa houve aumento dos acertos, evidenciando que a atividade contribui para o autocuidado no tratamento insulinoterápico.

Descritores: Diabetes Mellitus; Enfermagem; Insulina; Educação em Saúde; Estrutura de Grupo.

\section{ASBTRACT}

The objective of the present study was to analyze the skill that individuals with diabetes mellitus (DM) have in performing the insulin application process before and after and educational group session regarding this procedure. Data collection was performed through the application of a checklist that evaluated an individual's initial skill regarding the process. Next, an educational group session was held, and the same checklist was applied again to evaluate the individual's final skill. The data were stored in a databank and submitted to statistical analysis. The results showed that most participants had doubts in almost all stages of the insulin application process, and failed to perform some essential stages or simply skipped them. After the educational intervention, there was an increase in right procedures, which gives evidence that the activity contributes with self-care and with the insulin treatment.

Descriptors: Diabetes Mellitus; Nursing; Insulin; Health Education; Group Structure.

\section{RESUMEN}

El estudio apuntó a analizar la habilidad del afectado por diabetes mellitus (DM) para efectuar la aplicación de insulina antes y después de la enseñanza grupal del procedimiento. La recolección de datos se efectuó mediante un instrumento en forma de check list, que evaluó la habilidad inicial del paciente de DM en relación al proceso. Posteriormente, se realizó una intervención educativa grupal, aplicándose finalmente el mismo instrumento para evaluar la habilidad final. La información obtenida fue registrada en un banco de datos y sometida a análisis estadístico. Los resultados demostraron que la mayoría de las personas expresó dudas en casi todas las etapas del proceso de aplicación de insulina, pasando por alto algunas etapas esenciales o ejecutándolas de manera incorrecta. Luego de la intervención, existió aumento en los aciertos, poniéndose en evidencia que la actividad contribuye al autocuidado en el tratamiento insulinoterápico.

Descriptores: Diabetes Mellitus; Enfermería; Insulina; Educación en Salud; Estructura de Grupo. 


\section{INTRODUÇÃO}

As doenças crônicas não transmissíveis (DCNT) compõem um grupo de doenças que se caracterizam por apresentar longo período de latência, tempo de evolução prolongado, lesões irreversíveis e complicações que acarretam graus variáveis de incapacidade ou morte(1). $\mathrm{O}$ diabetes mellitus (DM) configura-se hoje como uma epidemia mundial(2), cujo impacto no Brasil foi revelado no registro nacional de diabetes e hipertensão, SisHiperdia iniciado em 2002, onde existem mais de 1,6 milhão de casos de DM registrados, e destes, 4,3\% tinham transtorno do pé diabético, 2,2\% amputação prévia, 7,8\% tinham doença renal e $7,8 \%$ haviam tido infarto do miocárdio(3).

Nessa direção, o DM é uma condição crônica que requer gerenciamento contínuo, sendo caracterizado por hiperglicemia e ocasionado por uma deficiência absoluta ou relativa de insulina, influenciando o metabolismo dos glicídios, proteínas, lipídios, água e vitaminas. De sua evolução, na dependência do controle metabólico, podem advir complicações agudas e crônicas micro e macrovasculares ${ }^{(1)}$. Dessa forma, o tratamento do DM inclui diversas estratégias que incluem a educação, modificação do estilo de vida e, se necessário, o uso de medicamentos $^{(4)}$. O uso da insulina, além da indicação clássica no DM1, está cada vez mais sendo indicado para as pessoas com DM2 e que, após seis anos de tratamento com sulfonilureias, 53\% das pessoas necessitaram do uso da insulina para obter um bom controle glicêmico ${ }^{(4)}$.

O objetivo da administração de insulina é aproximar o sujeito do perfil fisiológico da secreção pancreática de insulina normal. Dessa forma, múltiplas doses de insulina diárias no tecido subcutâneo podem ser necessárias no sentido de aperfeiçoar o controle glicêmico, o qual tem sido demonstrado como condição essencial na prevenção das complicações crônicas dessa doença ${ }^{(5)}$.

Um dos sérios problemas da insulinoterapia está relacionado à orientação e ao controle do medicamento que é realizado diariamente no domicílio, e em geral por familiares, à inconstância do horário dos medicamentos, à falta de seguimento da prescrição médica e a erros na administração da dose insulínica. Muitas vezes, as pessoas que têm DM possuem a ideia errônea de que o uso da insulina significa a falta de opção de tratamento e atribuem as complicações crônicas da doença aos efeitos colaterais da insulinoterapia, como também possuem medo do uso rotineiro de injeções subcutâneas e têm pouco conhecimento de como aplicar e realizar o armazenamento da insulina ${ }^{(6)}$.

Nessa direção, o processo de aplicação de insulina requer do sujeito conhecimento e habilidade técnica. Para que isso ocorra é necessário tempo, prática e educação continuada para que o usuário consiga desenvolver essa habilidade aliada à confiança(7). Cabe conhecer as estratégias de ensino na instrumentalização das pessoas para a auto aplicação de insulina. A estratégia de ensino pelo telefone evidenciou melhora na competência da pessoa com diabetes para o processo de aplicação de insulina ${ }^{(8)}$. Com as novas recomendações para aplicação de insulina estabelecidas nas diretrizes da Sociedade Brasileira de Diabetes ${ }^{(4)}$ outras estratégias de educação em saúde precisam ser testadas, e o presente estudo teve por objetivo analisar a habilidade da pessoa com DM em realizar o processo de aplicação de insulina antes e após o ensino em grupo desse procedimento.

\section{MÉTODOS}

Estudo do tipo antes e depois com estratégia de coleta e análise de dados quantitativa. Participaram os adultos com DM atendidos no Centro Educativo de Enfermagem para Adultos e Idosos, cadastrados no período de novembro de 2005 a novembro de 2007. Foram convidados por telefone 75 pessoas com DM que utilizavam insulina, com aceite de 19 pessoas em participar do estudo. Os motivos de recusa foram devido: 39 devido dificuldades de ordem pessoal, cinco financeira e 12 devido a própria condição de saúde.

Antes do início da coleta de dados, foi realizado um estudo piloto com três sujeitos que não foram incluídos na amostra, visando conhecer informações sobre recusas, perdas, a porcentagem de escolhas em cada um dos grupos e tempo gasto para o preenchimento dos instrumentos.

A coleta de dados ocorreu no período de outubro de 2010 a fevereiro de 2011. Foram configurados dois grupos, o primeiro contou com 11 pessoas e o segundo com oito participantes. Cada grupo de intervenção teve duração de um mês com quatro encontros semanais e duração média de duas horas cada encontro. A coordenação da atividade foi realizada pelos pesquisadores responsáveis. A atividade foi realizada no local do estudo como parte de um programa de educação 
em diabetes. Dentre as múltiplas propostas, abordagens e postulados teóricos disponíveis sobre grupos, adotouse a concepção do grupo de educação em saúde para otimizar o desenvolvimento grupal(9). Os grupos de autocuidado em DM, como o próprio nome indica, tem como objetivo melhorar o autocuidado, auxiliando as pessoas a adquirirem habilidades ou buscarem comportamentos mais saudáveis, que podem ser aprendidos ou aprimorados por meio do grupo. Essa estratégia é utilizada não apenas para oferecer informações que proporcionem atitudes e hábitos de vida saudável, como também para estimular a troca de experiências entre os participantes.

Os dados foram obtidos por meio da aplicação de um instrumento em forma de check list, que avaliou a habilidade inicial $(\mathrm{HI})$ da pessoa com DM em relação ao processo de aplicação de insulina. Posteriormente, realizou-se a intervenção educativa em grupo, norteada pelas dificuldades relacionadas ao processo de aplicação de insulina referido pelas pessoas com DM. Numa segunda fase, o mesmo questionário utilizado anteriormente foi aplicado novamente com o intuito de avaliar a habilidade final (HF) da pessoa com DM em relação ao processo de aplicação da insulina.

$\mathrm{O}$ instrumento de coleta de dados foi formulado e validado em um estudo sobre a utilização do telefone como estratégia de intervenção de enfermagem para o cuidado em DM, avaliando as habilidades necessárias a pessoa com DM para realização da aplicação de insulina no domicílio ${ }^{(8)}$. Ele utiliza variáveis sociodemográficas (sexo, idade, estado civil, escolaridade e ocupação), variáveis clínicas (tipo de diabetes, tempo de diagnóstico do diabetes, tempo de uso de insulina), variáveis antropométricas (peso, altura e índice de massa corporal) e variáveis relacionadas à administração de insulina (tipo de instrumento utilizado para a técnica, tipo de insulina, armazenamento e transporte, número de aplicações diárias, locais de aplicação, técnica de preparo e aplicação da insulina, profissional responsável pelo ensino prévio ao estudo, dificuldade na aquisição de insumos), que contemplam os objetivos do estudo.

Os dados obtidos foram para um banco de dados feito no programa Microsoft Excel, versão XP (Microsoft Co, USA), logo após, importados para o programa SPSS (Statistical Package for the Social Sciences) for Windows, versão 15.0 sendo submetidos à análise estatística. O projeto foi aprovado pelo Comitê de Ética em Pesquisa da Escola de Enfermagem de Ribeirão Preto, protocolo $1145 / 2010$, sendo que todos os participantes assinaram o Termo de Consentimento Livre e Esclarecido, permanecendo cada um com uma via.

\section{RESULTADOS}

Participaram do estudo 19 pessoas com DM que utilizavam insulina no Centro Educativo para Adultos e Idosos. A maioria foi representada pelo sexo masculino, que totalizou 13 (68,42\%) pessoas, nove (47,37\%) casados, quatro $(21,05 \%)$ com ensino médio completo e 11 (57,90\%) aposentados.

Com relação à idade, a média foi de 65 anos, sendo a maior idade de 75 anos e a menor de 59 anos. A maioria das pessoas $(57,89 \%)$ situou-se entre a faixa etária de 60 a 69 anos. Nas variáveis clínicas, notou-se que em relação ao tempo de diagnóstico do DM, o menor referenciado foi de cinco anos e o maior de 30 anos, sendo a média de 15 anos. Em termos de tempo de uso de insulina, o menor registrado foi de um ano e o maior de 30 anos, com média de nove anos. As variáveis antropométricas demonstraram que o maior peso encontrado foi de $89 \mathrm{~kg}$ e o menor de $54 \mathrm{~kg}$, com média de $69 \mathrm{~kg}$. Sobre a altura verificou-se como a maior de $172 \mathrm{~cm}$ e a menor de $144 \mathrm{~cm}$, com média de $159 \mathrm{~cm}$. Após esses resultados, foi calculado o índice de massa corporal (IMC), obtendo-se um valor máximo de $34 \mathrm{~kg} / \mathrm{m}^{2}$ e mínimo de $17 \mathrm{~kg} / \mathrm{m}^{2}$, com média de $24 \mathrm{~kg} / \mathrm{m}^{2}$.

A habilidade pessoa com DM em relação à aplicação de insulina foi avaliada por questões que permearam desde o tipo de insulina até a forma como esta é aplicada, além do modo como é realizado o armazenamento e descarte dos insumos utilizados. $O$ instrumento de coleta de dados foi aplicado em um primeiro momento denominando-se habilidade inicial $(\mathrm{HI})$, com posterior intervenção educativa e para verificação da eficiência da intervenção o instrumento foi aplicado novamente, denominando-se habilidade final (HF). Os resultados são apresentados na Tabela 1. 
Tabela 1. Tabela de frequência de acertos e erros para as variáveis propostas antes e após o ensino em grupo. Ribeirão Preto, SP, 2011.

\begin{tabular}{|c|c|c|c|c|c|}
\hline \multirow{3}{*}{ Variáveis } & \multirow{3}{*}{$\mathbf{n}$} & \multicolumn{2}{|c|}{ Habilidade Inicial HI } & \multicolumn{2}{|c|}{ Habilidade Final HF } \\
\hline & & Acertos & Erros & Acertos & Erros \\
\hline & & n (\%) & n (\%) & n (\%) & n (\%) \\
\hline Mistura de insulinas NPH e Regular & 19 & $17(89,47)$ & $2(10,53)$ & $19(100)$ & - \\
\hline Primeira insulina aspirada & 19 & $6(31,58)$ & $13(68,42)$ & $7(36,84)$ & $12(63,16)$ \\
\hline Seqüência da aspiração & 19 & $19(100)$ & - & $19(100)$ & - \\
\hline Tipo de instrumental & 19 & $14(73,68)$ & $5(26,32)$ & $18(94,74)$ & $1(5,26)$ \\
\hline Unidades de insulina & 19 & $16(84,21)$ & $3(15,79)$ & $19(100)$ & - \\
\hline Tamanho da seringa de insulina & 19 & $19(100)$ & $19(100)$ & $14(73,68)$ & $5(26,32)$ \\
\hline Tamanho de agulha & 19 & $17(89,47)$ & $2(10,53)$ & $18(94,74)$ & $1(5,26)$ \\
\hline Rodízio de aplicação de insulina & 19 & $11(57,89)$ & $8(42,11)$ & $19(100)$ & - \\
\hline Local para aplicação de insulina & 19 & $19(100)$ & - & $19(100)$ & - \\
\hline Armazenamento da insulina & 19 & $19(100)$ & - & $19(100)$ & - \\
\hline Orientação profissional & 19 & $17(89,47)$ & $2(10,53)$ & $19(100)$ & - \\
\hline Acondicionamento no gelo & 19 & $2(10,53)$ & $17(89,48)$ & $5(26,32)$ & $5(26,32)$ \\
\hline Tempo de exposição & 19 & $4(21,05)$ & $15(78,95)$ & $13(68,42)$ & $13(68,42)$ \\
\hline Lavagem das mãos & 19 & $19(100)$ & - & $19(100)$ & - \\
\hline Retirada da refrigeração & 19 & $10(52,63)$ & $9(47,37)$ & $16(84,21)$ & $3(15,79)$ \\
\hline Características da insulina & 19 & $19(100)$ & - & $19(100)$ & - \\
\hline Homogeneização da insulina & 19 & $13(68,42)$ & $6(31,58)$ & $17(89,47)$ & $2(10,53)$ \\
\hline Desinfecção da borracha do frasco & 19 & $11(57,89)$ & $8(42,11)$ & $15(78,95)$ & $4(21,06)$ \\
\hline Introdução de ar no frasco & 19 & $10(52,63)$ & $9(47,37)$ & $16(84,21)$ & $3(15,79)$ \\
\hline Posicionamento do frasco & 19 & $15(78,95)$ & $4(26,02)$ & $15(78,95)$ & $4(26,02)$ \\
\hline Retirada de bolhas de ar & 19 & $11(57,89)$ & $8(42,11)$ & $15(78,95)$ & $4(26,02)$ \\
\hline Ajuste da dose de insulina & 19 & $11(57,89)$ & $8(42,11)$ & $13(68,42)$ & $6(31,58)$ \\
\hline Reencape da agulha & 19 & $13(68,42)$ & $6(31,58)$ & $17(89,47)$ & $2(10,53)$ \\
\hline Antissepsia da pele & 19 & $17(89,47)$ & $2(10,53)$ & $19(100)$ & - \\
\hline Realizar a prega na pele & 19 & $15(78,95)$ & $4(21,05)$ & $17(89,47)$ & $2(10,53)$ \\
\hline Ângulo de introdução da agulha & 19 & $19(100)$ & - & $19(100)$ & - \\
\hline Aspiração da seringa & 19 & $4(21,05)$ & $15(78,95)$ & $13(68,42)$ & $6(31,58)$ \\
\hline Espera de 5 segundos & 19 & $6(31,58)$ & $13(68,42)$ & $17(89,47)$ & $2(10,53)$ \\
\hline Massagem no local da aplicação & 19 & $9(47,37)$ & $10(52,63)$ & $17(89,47)$ & $2(10,53)$ \\
\hline Armazenamento da insulina & 19 & $12(63,16)$ & $6(31,58)$ & $19(100)$ & - \\
\hline Tipo de transporte & 19 & $15(78,95)$ & $4(21,05)$ & $17(89,47)$ & $2(10,53)$ \\
\hline Acondicionamento & 19 & $14(73,68)$ & $5(26,32)$ & $19(100)$ & - \\
\hline
\end{tabular}

Após a análise das respostas obtidas, em relação ao conhecimento acerca do tipo de insulina, na HI 17 $(89,47 \%)$ pessoas acertaram a resposta e duas (10,53\%) erraram por não saberem referir o nome da insulina prescrita por seu médico. Após a intervenção, em HF observou-se que as duas pessoas passaram a acertar essa questão, pois souberam nominar corretamente o tipo de insulina que utilizam.

Quanto ao conhecimento do tamanho da seringa de insulina, na $\mathrm{HI} 14$ (73,68\%) pessoas responderam adequadamente e cinco (26,32\%) pessoas erraram, porém na avaliação da $\mathrm{HF}$, quatro $(21,05 \%)$ pessoas passaram a acertar a resposta, sendo que apenas uma $(5,26 \%)$ não obteve êxito quanto a resposta adequada.

Acerca da questão relacionada ao conhecimento da escala de graduação da seringa de insulina, a $\mathrm{HI}$ mostrou que 16 (84,21\%) pessoas procederam à resposta correta de 2 UI em relação ao uso da seringa de $100 \mathrm{Ul}$ e três $(15,79 \%)$ pessoas erraram. Na avaliação da HF, três $(15,79 \%)$ pessoas passaram a acertar essa questão. Sobre o conhecimento do tamanho da agulha nenhuma pessoa com DM entrevistada respondeu corretamente que a agulha deve ser proporcional ao IMC durante a $\mathrm{HI}$, no entanto, na HF 14 (73,68\%) pessoas responderam 
adequadamente e cinco (26,32\%) pessoas não obtiveram sucesso.

Com relação à avaliação da $\mathrm{HI}$ e HF sobre o conhecimento acerca do armazenamento da insulina, $100 \%$ das pessoas responderam corretamente que a mesma deve estar localizada na geladeira, nas gavetas de vegetais e nunca deve ser mantida na porta, conforme as recomendações da Sociedade Brasileira de Diabetes ${ }^{(9)}$.

Para a técnica de retirada do frasco de insulina da geladeira por alguns minutos antes da aplicação, na HI 10 (52,63\%) pessoas acertaram a resposta ao dizer que aguardam cinco minutos entre a retirada da insulina da geladeira e a aplicação, entretanto nove $(47,37 \%)$ pessoas erraram essa questão por não aguardarem ou por não terem conhecimento em relação a isso. Na avaliação da $\mathrm{HF}$, sete $(36,84 \%)$ pessoas passaram a realizar essa etapa e duas $(10,53 \%)$ pessoas permaneceram negligenciando este cuidado.

Sobre a etapa de observação das características da insulina antes de realizar a aspiração da mesma do frasco, $100 \%$ dos entrevistados acertaram a resposta tanto na $\mathrm{HI}$ quanto na $\mathrm{HF}$, pois confirmaram realizar esta etapa prévia a aspiração da insulina no domicílio. Com relação à desinfecção da borracha do frasco com álcool $70 \%$ antes de perfurá-la com a agulha, na HI 11 (57,89\%) pessoas declararam realizar essa etapa, seis $(31,58 \%)$ pessoas erraram essa pergunta por não realizarem e duas (10,53\%) pessoas não responderam a questão, ou seja, não são passíveis de erro ou acerto. Na HF quatro $(21,05 \%)$ pessoas passaram a realizar essa etapa e duas $(10,53 \%)$ pessoas permaneceram verbalizando que não a realizavam.

Para a etapa de introdução de ar no frasco de insulina para posterior aspiração, na HI 10 (52,63\%) pessoas acertaram ao mencionar que realizam tal procedimento e sete $(36,84 \%)$ pessoas erraram ao dizer que não realizam. Assim como na questão anterior, duas (10,53\%) pessoas não foram passíveis de erro ou acerto por não responderem à questão. $\mathrm{Na} \mathrm{HF}$, seis $(31,58 \%)$ pessoas passaram a acertar a questão por começarem a realizar a técnica e uma $(5,26 \%)$ pessoa ainda permaneceu errando a questão.

Acerca da questão sobre a realização do posicionamento do frasco de cabeça para baixo para a aspiração da insulina, na HI 15 (78,95\%) pessoas acertaram ao relatar que realizam essa etapa e duas
(10,53\%) pessoas erraram ao dizer que não a executavam. Novamente, duas $(10,53 \%)$ pessoas não responderam à questão, sendo classificadas como não passíveis de erro ou acerto. Na HF não houve acerto já que não existiu resposta para essa etapa.

A etapa que visa o golpeamento da seringa para a retirada de ar da mesma, obteve, na avaliação da HI, 11 $(57,89 \%)$ pessoas que acertaram a resposta por cumprirem esse passo antes da administração e seis $(31,58 \%)$ pessoas que erraram por não realizarem essa etapa. Novamente notou-se que duas (10,53\%) pessoas não responderam à questão, sendo classificadas como não passíveis de erro ou acerto. Na HF observou-se que cinco $(26,32)$ pessoas passaram a acertar a questão realizando a etapa e apenas uma (5,26\%) pessoa apresentou erro por não realizá-la.

Em relação ao ajuste da dose após a retirada de bolhas de ar, na HI 11 (57,89\%) pessoas mostraram que realizam esse processo, acertando a questão e seis (31,58\%) erraram por não o fazerem. Após, na avaliação da HF duas (10,53\%) pessoas passaram a realizar o ajuste com a resposta adequada e quatro (21,05\%) permaneceram não realizando a etapa, apresentando resposta inadequada. Nota-se também a ocorrência de duas (10,53\%) pessoas que não responderam e foram classificadas como não passíveis de acerto ou erro.

$\mathrm{Na}$ fase de avaliação da etapa de reencape da agulha, observou-se que na HI 13 (68,42\%) pessoas acertaram a resposta por procederem na realização desta etapa e seis $(31,58 \%)$ pessoas erraram por declararam que não realizam. Na HF essas seis (31,58\%) pessoas passaram a acertar a questão por realizarem a etapa. Sobre a antissepsia da pele, mostrou-se que $17(89,47)$ pessoas, na HI, acertaram a questão por realizarem essa etapa e duas (10,53\%) pessoas erraram por não a realizarem. Porém, na HF notou-se que essas duas (10,53\%) pessoas passaram a acertar a questão por começarem a realizar a técnica corretamente.

Em relação à realização da introdução da agulha na pele perpendicularmente, viu-se que $100 \%$ das pessoas acertaram a questão tanto na HI e HF por fazerem essa etapa corretamente. Já no que se diz respeito para a aspiração da seringa após a introdução da agulha, na HI quatro $(21,05 \%)$ pessoas acertaram a proposição e 15 (78,95\%) não obtiveram êxito na resposta. Рara a HF nove $(47,37 \%)$ pessoas responderam adequadamente e 
seis $(31,58 \%)$ pessoas ainda apresentaram erro na resposta.

Permeando a fase de aplicação da insulina que visa à espera de cinco segundos para a retirada da agulha, na $\mathrm{HI}$ seis $(31,58 \%)$ pessoas responderam corretamente por realizarem essa etapa, e 13 (68,42\%) pessoas declararam que não a realizam, errando, portanto a questão. Na HF, 11 (57,89\%) pessoas acertaram a proposição por realizarem o procedimento e apenas duas (10,53\%) pessoas ainda permaneceram apresentando erro na resposta por não efetuarem.

Quanto a massagearem o local da aplicação após a administração da insulina, a $\mathrm{HI}$ mostrou que nove $(47,37 \%)$ pessoas acertaram por dizerem não realizar essa etapa e 10 (52,63\%) pessoas erraram por verbalizar realizá-la. No entanto na $H F$, oito $(42,11 \%)$ pessoas passaram a obter sucesso na resposta, pois passaram a não realizá-la no domicílio e duas (10,53\%) pessoas permaneceram massageando o local da aplicação, desta forma apresentando uma prática inadequada a técnica.

O conhecimento do descarte do material perfurocortante mostrou na HI que 14 (73,68\%) pessoas acertaram a questão por descartarem as agulhas em locais adequados, tais como embalagens plásticas rígidas que após eram entregues em serviços de saúde. Em contrapartida, cinco (26,32\%) pessoas apresentaram erro em suas respostas ao referirem o descarte em locais inadequados. Posteriormente, na HF essas cinco (26,32\%) pessoas obtiveram sucesso na resposta, realizando o descarte correto do material.

\section{DISCUSSÃO}

Um dos marcos da terapêutica em DM ocorreu a partir de um estudo no qual se evidenciou que, ao ser submetido ao tratamento intensivo insulinoterápico, sob a orientação de uma equipe de educadores em DM, o indivíduo consegue manter os níveis de glicemia próximos à normalidade, além de prevenir as manifestações crônicas da doença(5). Para o alcance do controle glicêmico, a pessoa com DM deve ser orientada quanto ao preparo e realização correta da técnica de aplicação, segundo as recomendações da SBD ${ }^{(9)}$.

Anterior à realização da aplicação da insulina é de extrema importância que a pessoa tenha conhecimento sobre o tipo de insulina prescrita pelo seu médico, a medida da escala de graduação da seringa e o tamanho da agulha. Nesse sentido, o conhecimento em relação à escala de graduação da seringa se refere à legibilidade e clareza quanto ao número de unidades de insulina correspondentes a cada traço na seringa. Esta escala pode ser simples ou dupla, sendo que na primeira, cada traço da escala corresponde a uma unidade de insulina, presentes nas seringas de 30 UI e 50 UI. E na segunda a duas unidades de insulina, nas seringas de $100 \mathrm{UI}^{(9)}$.

No que diz respeito sobre o tamanho da agulha que deve ser utilizada estão disponíveis no mercado agulhas de diversos tamanhos e diâmetros, no entanto, quanto menor o diâmetro e comprimento da agulha menor será a dor durante a aplicação. Dessa forma, o tamanho da agulha deve ser proporcional ao IMC da pessoa(10).

Porém, na realização desse estudo tínhamos que agulhas mais curtas não são indicadas para pessoas obesas devido à possibilidade de variações na absorção da insulina ${ }^{(10-12)}$. No entanto, novas recomendações para a aplicação de insulina, recentemente divulgadas, apontam que as agulhas ultracurtas podem ser utilizadas pelas pessoas obesas, uma vez que, a camada dérmica não ultrapassa $0,4 \mathrm{~mm}^{(13)}$.

Agulhas com comprimentos anteriormente recomendadas para injeção de insulina no subcutâneo hoje são consideradas longas para adultos (por exemplo, agulhas de $12,7 \mathrm{~mm}$ ) e para a maioria das crianças (por exemplo, as agulhas de $8 \mathrm{~mm}$ ), pois elas aumentam o risco de injeção intramuscular. Assim, em crianças, adolescentes, adultos e obesos, os estudos têm confirmado a eficácia, a segurança e o menor desconforto com as agulhas de $4 \mathrm{~mm}, 5 \mathrm{~mm}$ e $6 \mathrm{~mm}^{(13)}$. Em contrapartida, o estudo de revisão(13) em questão ressalta seu uso para as pessoas que irão iniciar a aplicação de insulina, devido a redução do medo e ansiedade associado ao uso de uma agulha curta.

Em relação à técnica de aplicação de insulina deve-se destacar o fato de que antes da administração é de extrema importância a visualização das características da insulina no intuito de observar sinais de aquecimento, congelamento, precipitação ou mudança na cor, o que pode significar diminuição da ação da insulina ${ }^{(9)}$. Na presença dessas alterações, o frasco deve ser inutilizado e descartado. Dessa forma, este estudo observou que a totalidade das pessoas realizam corretamente essa etapa, corroborando com achados de outros estudos ${ }^{(8,14-15)}$. 
Sobre a etapa de desinfecção da borracha do frasco de insulina com álcool $70 \%$ foi possível identificar que $57,89 \%$ das pessoas a realizavam antes da intervenção em grupo, sendo que posteriormente a esta, 78,94\% passaram a realizá-la. Outro estudo realizado com crianças e adolescentes, obteve-se que $94,5 \%$ da população estudada realizava a desinfecção com algum tipo de álcool, sendo que o mais utilizado foi o álcool de supermercado ${ }^{(16)}$. Em um estudo referente aos usuários atendidos na Estratégia Saúde da Família (ESF), 59,8\% referiram realizar a etapa, porém não foi encontrado relato de uso de álcool $70 \%$, sendo o álcool de supermercado utilizado pela totalidade da população(14). Em relação aos profissionais de saúde, o estudo mostrou que todos eles sempre executaram a desinfecção com álcool $70 \%{ }^{(15)}$.

Para a etapa de preparo da dose de insulina a ser administrada o presente trabalho encontrou que 52,63\% das pessoas antes da intervenção em grupo realizavam a introdução de ar no frasco de insulina e 57,89\%, após a aspiração da insulina do frasco, realizavam o golpeamento da seringa para a retirada de ar e ajustavam novamente a dose de insulina após a saída das bolhas de аг. Posteriormente a intervenção, $84,21 \%$ das pessoas passaram a introduzir ar dentro do frasco de insulina e a golpear a seringa para a retirada das bolhas de ar. Notouse também que $68,42 \%$ passaram a ajustar a dose de insulina novamente depois da retirada do ar. Destacam-se as recomendações da Sociedade Brasileira de Diabetes pertinentes a essa questão(9).

As pequenas bolhas de ar presentes na seringa não causam prejuízos se injetadas, porém a presença das mesmas faz com que haja uma redução da quantidade de insulina prescrita. Assim, no estudo realizado com usuários da ESF foi possível perceber que 90,5\% deles relatam eliminar as bolhas de $a r^{(14)}$. No entanto, em relação aos profissionais de saúde tornou-se preocupante o fato de que em $12,2 \%$ dos casos houve prejuízo da dose de insulina pela não retirada das bolhas de $a r^{(15)}$.

Albergando as questões norteadoras sobre a técnica de aplicação de insulina, o atual trabalho mostrou que anteriormente ao ensino em grupo $89,47 \%$ das pessoas realizavam a antissepsia da pele para a aplicação de insulina, sendo que após o ensino, 100\% das pessoas passaram a realizar essa etapa. No que se refere aos profissionais de saúde, um estudo mostrou que $97,2 \%$ realizaram a antissepsia da pele adequadamente antes da introdução da agulha na pele ${ }^{(15)}$. Tratando-se do estudo com crianças e adolescentes, percebeu-se que $96 \%$ realizaram a antissepsia da pele, sendo que a maioria $(54,3 \%)$ utilizou o álcool de supermercado. Foi encontrado também a utilização de outros tipos de soluções para a antissepsia da pele, como água e sabão, soro fisiológico e bicarbonato de sódio(16).

Sobre o modo de inserção da agulha na pele para a administração da insulina, encontrou-se que $100 \%$ das pessoas introduziam a agulha de forma perpendicular à pele, num ângulo de 90 graus, corroborando com o preconizado pela SBD ${ }^{(10)}$, no momento da realização do estudo. Este fato foi identificado antes e após o ensino em grupo. No entanto, faz-se necessário destacar que a angulação da agulha está relacionada atualmente com o tamanho da agulha, além de considerar aspectos físicos, psicológicos e a técnica de aplicação(9). Nessa direção, anteriormente a angulação da agulha estava estritamente relacionada ao $I M C^{(10)}$, porém, diante de estudos recentes ${ }^{(13)}$, a SBD(9) recomenda que agulhas longas, de $12,7 \mathrm{~mm}$ devam ser utilizadas exclusivamente para adultos obesos ou com sobrepeso, onde o profissional de saúde deve avaliar e orientar a utilização de ângulo diferente de 90 graus para evitar a aplicação via intramuscular da insulina.

Um fator importante após a administração da insulina reside na compressão do local de aplicação por cinco a oito segundos. A compressão do local auxilia na diminuição do extravasamento de insulina para fora do tecido subcutâneo. Entretanto, chama-se a atenção de que este estudo identificou que $47,37 \%$ das pessoas realizavam a massagem do local de aplicação antes da atividade em grupo e que posteriormente a isso, 89,48\% passaram a não realizá-la. Massagear o local de aplicação pode alterar o tempo de ação da insulina, assim é preciso que os profissionais de saúde estejam atentos durante as orientações, dando-se ênfase na compressão do local e não em massageá-lo( ${ }^{(9,13)}$.

No que se refere ao descarte correto do material utilizado, é importante destacar que muitas vezes a pessoa com DM não recebe orientações quanto ao procedimento adequado, descartando muitas vezes agulhas e seringas em lixo domiciliar, conforme referido por cinco $(26,32 \%)$ pessoas neste estudo antes do ensino em grupo. 


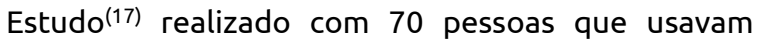
insulina atendidas no ambulatório de Endocrinologia de um hospital-escola do município de Campinas, São Paulo, revelou resultados alarmantes, nos quais $100 \%$ dos entrevistados não souberam diferenciar lixo doméstico do biológico e $51,43 \%$ descartavam no lixo comum as agulhas e seringas, demonstrando a importância da conscientização e educação da população quanto aos riscos desta prática como fundamental para o meio ambiente. Portanto, o autor ressalta que as Unidades Básicas de Saúde deverão propiciar aos sujeitos da sua abrangência, o manejo adequado dos resíduos, demonstrando que o descarte inadequado de seringas e agulhas proveniente de práticas equivocadas, pode ameaçar a qualidade de vida.

Em relação à higienização das mãos, condição necessária para evitar contaminação dos materiais e diminuição da ocorrência de infecções nos locais de

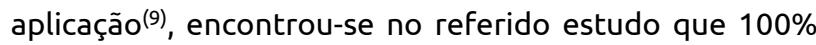
das pessoas referiram realizar a lavagem das mãos antes da execução do processo, antes e após o ensino em grupo. Entre os usuários da ESF ficou demonstrado que $88,8 \%$ também a realizavam, utilizando água e sabão ou detergente líquido ${ }^{(14)}$. Entre os profissionais de saúde, o estudo concluiu que $73,2 \%$ destes deixaram de realizar a higienização das mãos, fato que chama à atenção na medida em que sãos os próprios profissionais de saúde, principalmente de enfermagem, que atuam como modelos para a orientação do processo de aplicação de insulina ${ }^{(15)}$.

Quanto à realização dos rodízios entre os locais de aplicação, no intuito de evitar complicações como lipodistrofia insulínica, lipo-hipertrofia, hematomas, nódulos endurecidos, infecções, ente outros, os achados demonstraram que anteriormente ao ensino em grupo apenas $57,89 \%$ das pessoas alternavam os locais de aplicação, porém após a atividade em grupo, 100\% das pessoas passaram a realizar. É considerável dizer que entre os profissionais de saúde o estudo mostrou que em $85,4 \%$ dos procedimentos de aplicação de insulina, os mesmos não consideraram o local da última aplicação(15).

Dessa forma, faz-se necessário a capacitação dos profissionais de saúde na orientação do processo de aplicação de insulina, já que estes são os principais orientadores do cuidado.
Especialmente na área da saúde, na qual o grupo é uma presença constante, tanto no convívio da equipe de trabalho, como ferramenta terapêutica ou de capacitação e desenvolvimento de pessoas, é fundamental a qualificação de profissionais mais aptos a lidar com os fenômenos grupais, de modo a ter um manejo embasado em referencial teórico-prático consolidado(18). Dentre estes profissionais, chama-se a atenção para o enfermeiro, modelo da equipe de enfermagem e peçachave na promoção do autocuidado nos serviços de saúde.

\section{CONCLUSÕES}

O presente estudo permitiu identificar que as pessoas com DM que fazem uso da terapia insulínica apresentam erros em diversas etapas do processo de aplicação de insulina. Os dados mostram que, com a intervenção proposta, foi possível avaliar que, após o ensino em grupo, as etapas do processo de aplicação passaram a ser referidas corretamente por grande parte das pessoas. Dessa forma, a intervenção em grupo pode contribuir para a promoção da educação em DM e para a melhora e eficácia do tratamento insulínico, podendo ser utilizada como estratégia para incrementar a competência da pessoa com diabetes para aplicação de insulina. O conhecimento advindo deste estudo oferece subsídios valiosos para incrementar a observância do plano terapêutico. Espera-se que esse conhecimento possa ter impacto sobre as práticas em saúde.

O apoio para auto-aplicação da insulina é um componente essencial no cuidado a pessoas com doenças crônicas, como o diabetes. Este estudo mostrou que é possível aprimorar o empoderamento do paciente para aplicação da insulina por meio de estratégia em grupo. O êxito dessa empreitada depende, em larga medida, do conhecimento e das habilidades que podem ser potencializadas a partir do encontro entre a equipe preparada e proativa e pacientes que se tornam bem informados e "empoderados" para enfrentar os desafios inerentes a essa tarefa.

Algumas limitações puderam ser observadas, decorrentes do desenho metodológico utilizado no presente estudo, que não permite a generalização dos resultados. Assim, são necessárias novas investigações, com estratégias metodológicas mais robustas e amostras maiores. 
Os profissionais envolvidos com a educação em DM devem manter-se em constante atualização para garantir uma assistência de qualidade, favorecendo a prática de

\section{REFERÊNCIAS}

1. American Diabetes Association. Diagnosis and classification of diabetes mellitus. Diabetes Care. 2010; 34 Suppl 1:S62-S69. 2. Shaw JE, Sicree RA, Zimmet PZ. Global estimates of the prevalence of diabetes for 2010 and 2030. Diabetes Res Clin Pract. 2010; 87(1):4-14.

3. Schmidt MI, Duncan BB, Silva GA, Menezes AM, Monteiro CA, Barreto SM, et al. Chronic non-communicable diseases in Brazil: burden and current challenges. Lancet. 2011;377:1949-61. 4. Sociedade Brasileira de Diabetes. Diretrizes da Sociedade Brasileira de Diabetes. Londrina (Brasil), 2011. 340p.

5. United Kingdom Prospective Diabetes Study Group. Intensive blood-glucose control with sulfonylureas or insulin compared with conventional treatment and risk of complications in patients with type 2 diabetes: UKPDS 33. Lancet, New York. 1998;352:837-53.

6. Bonifacio NA, Michelin AF, Costa LRLG, Zanetti ML, Cassiani $\mathrm{SHB}$, Teixeira CRS. Processo de administração de insulina subcutânea em pacientes diabéticos hospitalizados. Ciênc. Cuidado Saúde, Maringá: 2008;7(2):171-179.

7. Teixeira CRS, Zanetti ML, Pereira MCA. Perfil de diagnósticos de enfermagem em pessoas com diabetes segundo modelo conceitual de Orem. Acta Paul Enferm. 2009;22(4)385:91. 8. Becker TAC, Teixeira CRS, Zanetti ML. O acompanhamento por telefone como intervenção de enfermagem na aplicação de insulina. Acta Paul Enferm. 2012. No prelo.

9. Dias VP, Silveira DT, Witt RR. Educação em saúde: o trabalho de grupos em atenção primária Rev. APS, 2009 (12) 2:221-227. 10. Sociedade Brasileira de Diabetes. Tratamento e acompanhamento do Diabetes Mellitus. Diretrizes da Sociedade Brasileira de Diabetes. Rio de Janeiro: Sociedade Brasileira de Diabetes; 2007. 168 p.

11. American Diabetes Association. Insulin administration. Diabetes Care. 2004;27(Suppl):106-9.

12. Teixeira CRS, Zanetti ML. Administração de insulina: Uma abordagem fundamental na educação em diabetes. Rev Esc Enferm USP. 2000;34(3):264-70.

13. Frid A, Hirsch L, Gaspar R, Hicks D, Kreugel G, Liersch J et al. New injection recommendations for patients with diabetes. Diabetes Metab. 2010;36 Suppl 2:S3-18.

14. Stacciarini TSG, Pace AE, HAAS VJ. Técnica de autoaplicação de insulina com seringas descartáveis entre os usuários com diabetes mellitus, acompanhados pela Estratégia Saúde da Família. Rev. Latino-am Enfermagem. 2009;17(4):474-480. 15. Bonifácio NA, Michelin AF, Costa LRLG, Zanetti ML, Cassiani SHB, Teixeira, CRS. Processo de administração de insulina subcutânea em pacientes diabéticos hospitalizados. Cien.Cuid. Saúde. 2008;7(2):171-79.

16. Castro ARV, Grossi SAA. Reutilização de seringas descartáveis no domicílio de crianças e adolescentes com diabetes mellitus. Rev. Esc. Enferm. USP. 2007;41(2):187-95. 17. Tapia CEV. Diabetes mellitus e o descarte de seringas e agulhas. Rev Gaucha Enferm., 2009;30(2):228-34.

18. Motta KAMB, Munari DB, Leal ML, Medeiros M, Nunes FC. As trilhas essenciais que fundamentam o processo e desenvolvimento da dinâmica grupal. Rev. Eletr. Enf. [Internet] autocuidado e auto-aplicação de insulina, principalmente após as novas recomendações para aplicação de insulina de pessoas com DM.

2007 [acesso em: 29 mar 2013];9(1):229-41. Available from: http://www.fen.ufg.br/revista/v9/n1/v9n1a18.htm.

Artigo recebido em 06/11/2011. Aprovado para publicação em 12/06/2012. Artigo publicado em 31/03/2013. 